\title{
A nanocompartment containing the peroxidase DypB contributes to defense against oxidative stress in $M$. tuberculosis
}

Katie A. Lien¹, Robert J. Nichols², Caleb Cassidy-Amstutz², Kayla Dinshaw¹, Matthew Knight ${ }^{3}$, Rahul Singh4, Lindsay D. Eltis", David F. Savage², Sarah A. Stanley ${ }^{1,5, \#}$

1Department of Molecular and Cell Biology, Division of Immunology and Pathogenesis, University of California, Berkeley

2Department of Molecular and Cell Biology, Division of Biochemistry, Biophysics and Structural Biology, University of California, Berkeley

3Department of Plant and Microbial Biology, University of California, Berkeley

4Department of Microbiology and Immunology, The University of British Columbia, Vancouver, Canada ${ }^{5}$ School of Public Health, Division of Infectious Diseases and Vaccinology, University of California, Berkeley

\#corresponding author 


\begin{abstract}
Encapsulin nanocompartments are an emerging class of prokaryotic protein-based organelles consisting of an encapsulin protein shell that encloses a protein cargo'. Genes encoding nanocompartments are widespread in bacteria and archaea, and recent works have characterized the biochemical function of several cargo enzymes². However, the importance of these organelles to host physiology is poorly understood. Here, we report that the human pathogen Mycobacterium tuberculosis (Mtb) produces a nanocompartment that contains the dye-decolorizing peroxidase DypB. We show that this nanocompartment is important for the ability of Mtb to resist oxidative stress in low pH environments, including during infection of host cells and upon treatment with a clinically relevant antibiotic. Our findings are the first to implicate a nanocompartment in bacterial pathogenesis and reveal a new mechanism that Mtb uses to combat oxidative stress.
\end{abstract}


Results. Bacterial cells were long thought to lack compartmentalization of function. However, the identification of organelle-like structures including microcompartments, anammoxosomes, magnetosomes and, most recently, encapsulin nanocompartments have revolutionized our understanding of bacterial cell biology ${ }^{3-5}$. Characterized nanocompartments are proteinaceous shells that are 24 to $45 \mathrm{~nm}$ in diameter and comprised of $60-240$ subunits of a single protomer ${ }^{1,6}$. This shell surrounds ("encapsulates”) an enzymatic cargo protein'. Although putative encapsulin systems have been identified in $>900$ bacterial and archaeal genomes ${ }^{2,7}$, very little is known about their physiological function. Based on genomic organization, encapsulin systems are often predicted to compartmentalize enzymes involved in oxidative stress defense, iron storage, and anaerobic ammonium oxidation ${ }^{2}$. However, the function of an encapsulin system has only ever been demonstrated in a single bacterial species, Myxococcus xanthus ${ }^{8}$, and the physiological role of nanocompartments is largely unexplored.

The Mycobacterium tuberculosis genome encodes the predicted encapsulin gene $R v 0798 c /$ Cfp299 $^{9}$ in a two-gene operon with $R v 0799 c$, the dye-decolorizing peroxidase DypB (Figure 1A). Overexpression of the predicted Mtb encapsulin gene in Escherichia coli was previously shown to result in the formation of nanocompartment-like structures ${ }^{9}$. Three potential cargo proteins for the nanocompartment were proposed based on a putative shared encapsulation targeting sequence: DypB, FolB, and BrfB. In E. coli, overexpression of each protein with Cfp29 resulted in encapsulation. However, this study did not address whether Mtb produces endogenous nanocompartments or identify the specific function of these compartments in Mtb biology. A transposon screen has identified Cfp29 as a gene required for growth in mice ${ }^{10}$ and Cfp29 has long been known as an immunodominant T cell antigen in both mice and human TB patients ${ }^{11}$. Taken together, these results suggest that Mtb may produce an encapsulin nanocompartment that is important for infection. 
A

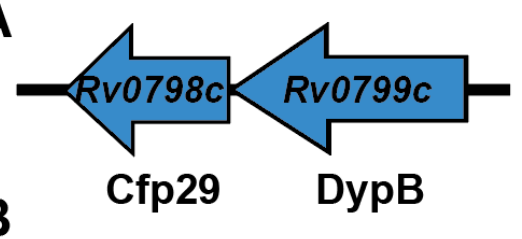

B
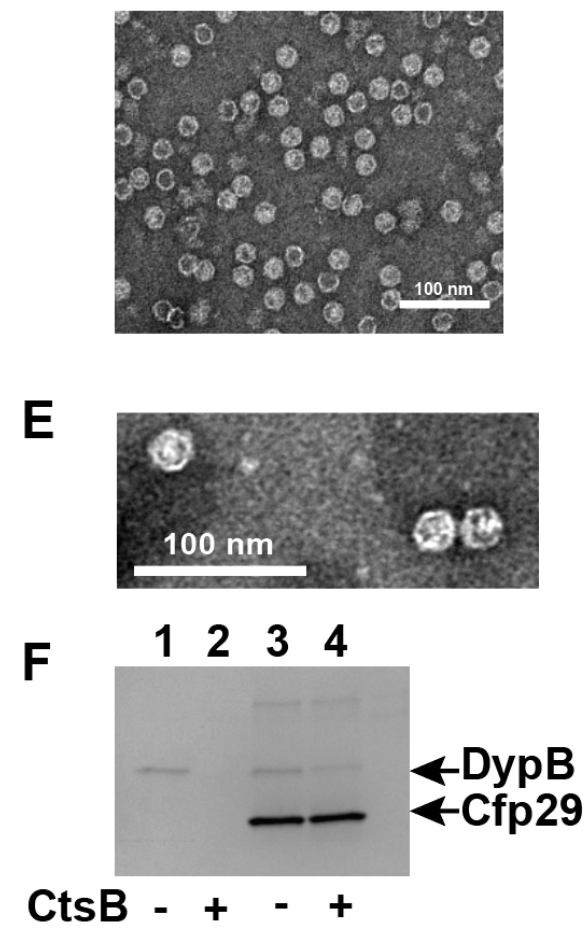

C

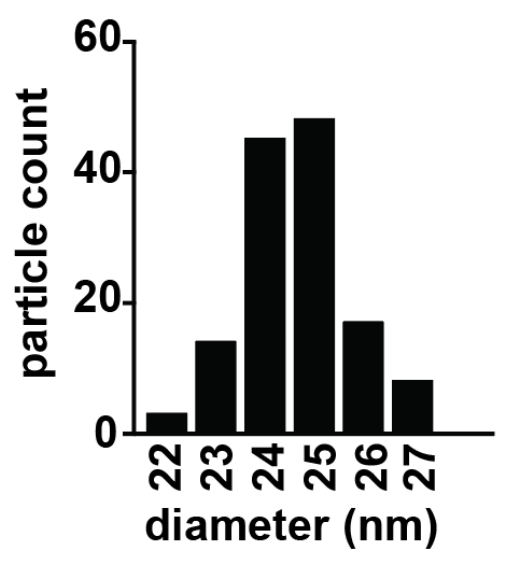

D

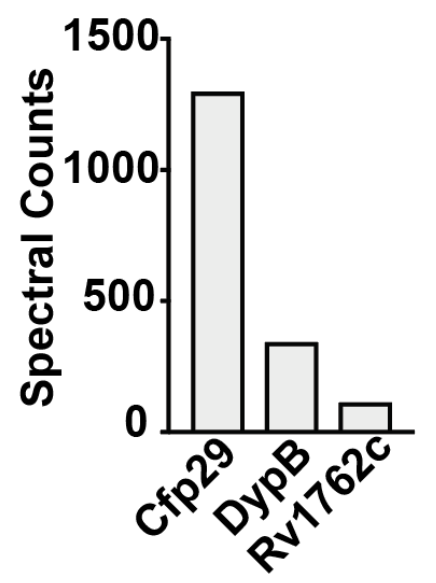

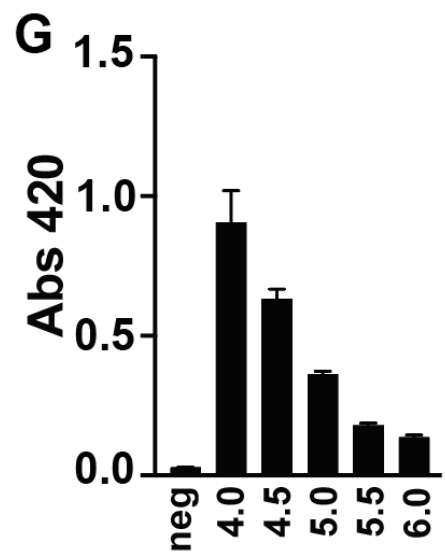

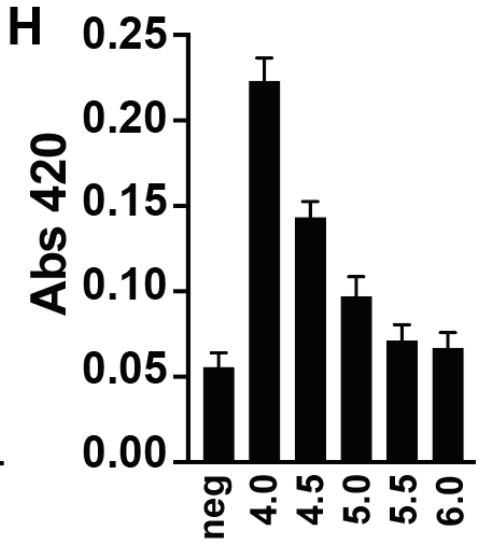

Figure 1. Mtb produces endogenous nanocompartments that package a peroxidase. (A) Schematic of the nanocompartment operon in Mtb which encodes the encapsulin shell protein (Cfp29) and the dye-decoloring peroxidase cargo protein (DypB). (B) TEM of Cfp29 encapsulin proteins purified following heterologous expression of the Mtb nanocompartment operon in E. coli. (C) Size distribution of Cfp29 protomers purified from E. coli. (D) Peptide counts from mass spectrometry analysis of endogenous nanocompartments purified from Mtb. (E) TEM of endogenous nanocompartments purified from Mtb. (F) Coomassie-stained SDS-PAGE of unencapsulated (lanes 1 and 2) and encapsulated (lanes 3 and 4) DypB following 14 hours of Cathepsin B (CtsB) treatment. Peroxidase activity of (G) unencapsulated and $(\mathrm{H})$ encapsulated DypB $(5 \mathrm{nM})$ using ABTS $(480 \mathrm{nM})$ as a substrate in the presence of $\mathrm{H}_{2} \mathrm{O}_{2}(480 \mathrm{nM})$ at varying pH levels $(4.0-6.0)$ as reported by a change in the absorbance at $420 \mathrm{~nm}$. neg=no added enzyme.

To confirm the previous finding that heterologous expression of Rv0798c and Rv0799c in a host species results in the assembly of an encapsulin system, we expressed these genes in E. coli and isolated nanocompartments. Clarified protein lysates from E. coli were purified by ultracentrifugation and size exclusion chromatography. Assembled encapsulin 
nanocompartments are distinguishable by their high molecular weight ${ }^{10}$. Indeed, a fraction from the purification contained a high-molecular weight species $>260 \mathrm{kDa}$ observable on an SDSPAGE gel (Figure S1A). Fractions containing putative nanocompartments were pooled and imaged using transmission electron microscopy, which revealed the presence of nanocompartment-like icosahedral structures with the expected diameter of $\sim 25 \mathrm{~nm}$ (Figure 1B, $1 \mathrm{C}$.

To determine whether Mtb produces nanocompartments under normal laboratory growth conditions, we performed an ultracentrifugation-based nanocompartment isolation strategy using wild-type H37Rv strain bacteria grown to mid-log phase (Figure S1B). Mass spectrometry analysis of the nanocompartment fraction identified both the encapsulin protein Cfp29 and the peroxidase DypB (Figure 1D). TEM analysis confirmed the presence of nanocompartment particles $\sim 25 \mathrm{~nm}$ in diameter (Figure 1E). Interestingly, Rv1762c, a protein of unknown function, was consistently identified in purified nanocompartment preparations from Mtb (Figure 1D). We were unable to identify either FolB or BrfB in nanocompartments from Mtb, suggesting that these proteins are not endogenous substrates for encapsulation under normal laboratory growth conditions for Mtb.

Cfp29 ('culture filtrate protein 29') was originally identified in the supernatants of Mtb cells grown in axenic culture ${ }^{12}$. As Cfp29 lacks a secretory signal sequence and is part of a large macromolecular complex, it is unclear how a nanocompartment could be actively secreted. However, nanocompartment structures are remarkably stable ${ }^{13}$ and it is possible that nanocompartments released from dying bacteria accumulate in culture as they are highly resistant to proteolysis/degradation. To test this hypothesis, we purified unencapsulated DypB and encapsulated DypB and exposed them to a lysosomal protease, cathepsin B, while monitoring proteolysis. Whereas unencapsulated DypB was completely degraded after 14 hours of exposure 
to cathepsin B, encapsulated DypB was protected from degradation, demonstrating the resistance of nanocompartment structures to proteolysis (Figure 1F).

DypB proteins are known to have low $\mathrm{pH}$ optima ${ }^{9,14}$. To demonstrate that Mtb DypB has a similarly low pH optimum, SUMO-tagged unencapsulated DypB was purified from E. coli (Figure S2A), and the peroxidase activity was evaluated at a range of $\mathrm{pH}$ values using the ABTS $(2,2$ '-azinobis(3-ethylbenzothiazoline-6-sulfonic acid)) dye-decolorizing assay previously used to characterize DypB proteins ${ }^{9,15}$. Similar to the Vibrio cholerae DypB ${ }^{14}$, purified Mtb DypB had increased enzymatic activity in low $\mathrm{pH}$ environments, with the greatest efficacy at $\mathrm{pH} 4.0$, the lowest $\mathrm{pH}$ tested (Figure $1 \mathrm{G}$ ). We next tested the ability of encapsulated DypB to degrade ABTS across a range of $\mathrm{pH}$ values. Similar to free $\mathrm{DypB}$, the encapsulated enzyme had the highest activity at $\mathrm{pH} \sim 4.0$ (Figure $1 \mathrm{H}$ ). Taken together, these data demonstrate the stability and functionality of $M$. tuberculosis nanocompartments under proteolytic and acid stress, conditions that mimic the host lysosomal environment.

The Mtb DypB protein was previously shown to function as a bona fide dye-decolorizing peroxidase ${ }^{9}$. Because peroxidases consume $\mathrm{H}_{2} \mathrm{O}_{2}$, they often participate in defense against oxidative stress ${ }^{16}$. During macrophage infection, the phagocytes initiate an oxidative burst that exposes Mtb to $\mathrm{H}_{2} \mathrm{O}_{2}{ }^{17}$. We therefore reasoned that DypB-containing nanocompartments may function to protect Mtb from $\mathrm{H}_{2} \mathrm{O}_{2}$-induced stress. To test this hypothesis, we created a mutant strain lacking both genes from the nanocompartment operon (Figure 1A, $\Delta$ operon). Lysates from $\Delta$ operon mutants were used for nanocompartment purification (data not shown) and western blot analysis using an antibody for Cfp29 as a probe (Figure S1C). Data from these analyses revealed that $\Delta$ operon mutants cannot produce viable nanocompartments. In addition, we isolated a 
A

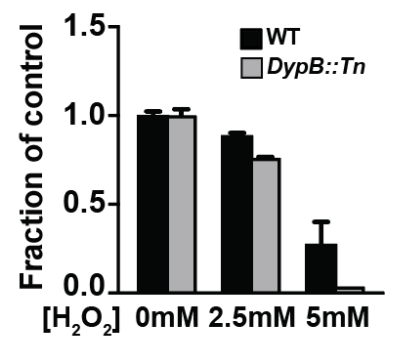

D

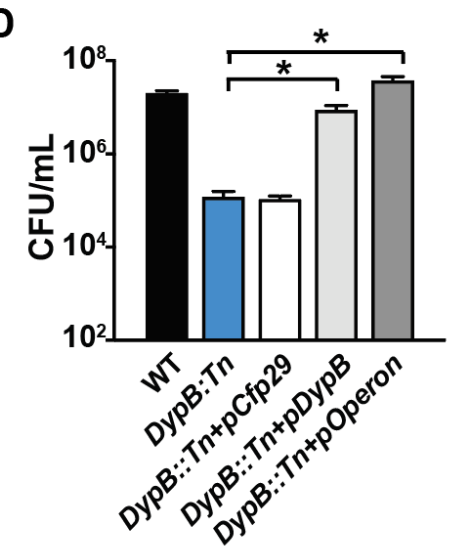

B

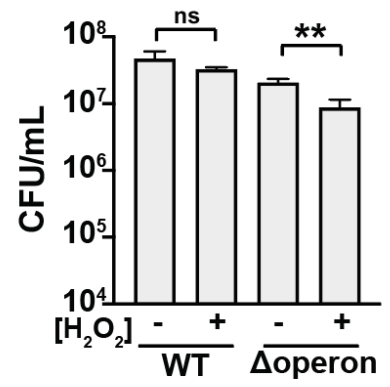

E

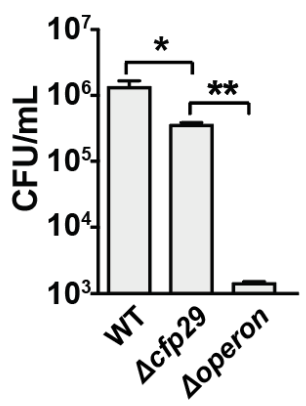

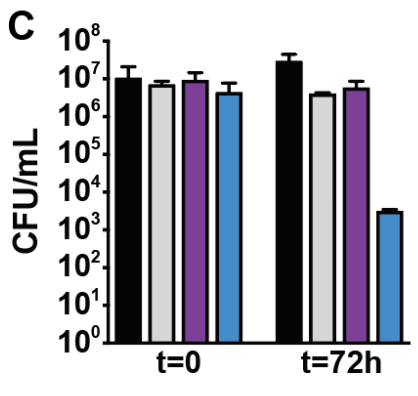

$\mathbf{F}$

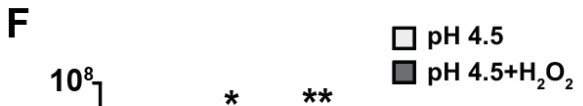

Figure 2. Nanocompartments protect Mtb from oxidative stress in acidic environments. (A) $\mathrm{OD}_{600}$ measurements of wild-type and DypB::Tn Mtb grown in 7H9 medium following exposure to $\mathrm{H}_{2} \mathrm{O}_{2}$ for 96 hours. Values reported are normalized to the untreated controls. CFU enumeration of wild-type and Mtb nanocompartment mutants grown in (B) standard 7H9 medium ( $\mathrm{pH} 6.5)$ and (C, E, F) acidified $7 \mathrm{H} 9$ medium $(\mathrm{pH} \mathrm{4.5)} \mathrm{following}$ exposure to oxidative stress $\left(2.5 \mathrm{mM} \mathrm{H}_{2} \mathrm{O}_{2}\right)$ for 72 hours. (D) CFU enumeration of wild-type, DypB::Tn mutants, and complemented mutants (pCfp29, pDypB, pOperon) following 24 hour exposure to oxidative stress $\left(2.5 \mathrm{mM} \mathrm{H}_{2} \mathrm{O}_{2}\right)$ in acidified $7 \mathrm{H} 9$ medium $(\mathrm{pH} 4.5)$. Figures are representative of at least $2(E, F)$ or $3(A-D)$ independent experiments. p-values were determined using unpaired t test. ${ }^{*} p<0.05,{ }^{* *} p<0.01$.

transposon mutant $(D y p B:: T n)$ containing an insertion in $D y p B$, a mutation that eliminated expression of both Cfp29 and DypB (Figure S1D).

To test whether DypB-containing nanocompartments are required for defense against $\mathrm{H}_{2} \mathrm{O}_{2}$, we exposed wild-type and DypB::Tn Mtb to increasing concentrations of $\mathrm{H}_{2} \mathrm{O}_{2}$ and monitored bacterial survival. Mutants lacking DypB nanocompartments were more susceptible to $\mathrm{H}_{2} \mathrm{O}_{2}$ when compared with wild-type bacteria as measured by $\mathrm{OD}_{600}$ (Figure $2 \mathrm{~A}$ ) and by plating for CFU 
(Figure 2B). However, the phenotype was relatively modest. We next reasoned that DypB nanocompartments might be required to resist oxidative stress in acidic environments that more closely mimic the in vivo environment. During infection, Mtb bacilli encounter the low $\mathrm{pH}$ of the phagolysosome, and the ability to tolerate low $\mathrm{pH}$ is required for Mtb survival in both infected macrophages and mice ${ }^{18}$. We therefore tested the susceptibility of DypB mutants to a combination of $\mathrm{H}_{2} \mathrm{O}_{2}$ and acid stress ( $\mathrm{pH}$ 4.5). Wild-type Mtb was able to withstand these conditions and did not significantly decrease in number over a three-day exposure period (Figure 2C). In contrast, the $\Delta$ operon mutant was resistant to each stressor individually, but was highly susceptible to $\mathrm{H}_{2} \mathrm{O}_{2}$ at $\mathrm{pH} 4.5$ (Figure 2C). Thus, DypB-containing nanocompartments are required to protect bacteria from oxidative stress at low $\mathrm{pH}$.

We next sought to determine whether encapsulation of DypB is important for its activity in bacterial cells. To accomplish this, DypB::Tn mutants were complemented with constructs expressing DypB alone (pDypB), Cfp29 encapsulin alone (pCfp29), or both proteins (pOperon). As expected, expression of DypB did not restore production of nanocompartments, whereas expression of Cfp29 alone resulted in the formation of empty nanocompartment structures (Figure S1D). Coexpression of both proteins resulted in the formation of DypB-containing nanocompartments in the DypB::Tn mutant (Figure S1D). We exposed the full set of complemented mutants to $\mathrm{H}_{2} \mathrm{O}_{2}$ at $\mathrm{pH} 4.5$ and determined survival after three days by plating for CFU. As expected, DypB::Tn was attenuated for survival under these conditions when compared to wild-type bacteria (Figure 2D). Restoring expression of the nanocompartment shell protein in the absence of DypB had no effect on bacterial survival (Figure 2D). Complementation by overexpression of unencapsulated DypB was sufficient to confer almost wild-type levels of resistance to oxidative and acid stress (Figure 2D). However, complementation with both Cfp29 and DypB resulted in enhanced resistance to 
these stressors when compared to mutants complemented with DypB alone (Figure 2B), suggesting that encapsulation of DypB enhances its function.

In the mutant complemented with DypB alone, it is possible that overexpression of the peroxidase compensates for the lack of encapsulation. We therefore examined the importance of DypB encapsulation in mutant strains in which we deleted Cfp29 only ( $\Delta$ cfp29). Deletion of Cfp29 resulted in a $\sim 0.5$ log decrease in bacterial viability after three days of exposure to $\mathrm{H}_{2} \mathrm{O}_{2}$ at $\mathrm{pH}$ 4.5, confirming that encapsulation of the peroxidase in the shell protein is important for full protection (Figure 2E). As expected, greater attenuation was observed in the mutant lacking both DypB and Cfp29 (Loperon). Our mass spectrometry data suggested that an additional protein, Rv1762c, associates with the Mtb encapsulin nanocompartment (Figure 1E). To test whether Rv1762c has functional significance, we isolated a mutant with a transposon insertion in this gene ( $R v 1762 c:: T n)$ and exposed it to $\mathrm{H}_{2} \mathrm{O}_{2}$ at $\mathrm{pH}$ 4.5. We found that $R v 1762 c:: T n$ is moderately susceptible to a combination of low $\mathrm{pH}$ and $\mathrm{H}_{2} \mathrm{O}_{2}$ (Figure 2F). Taken together, these data demonstrate that encapsulation of DypB enhances protection against oxidative stress in low $\mathrm{pH}$ conditions and that Rv1762c may have a functional role in the DypB encapsulin system.

The Mtb genome encodes $~ 18$ putative peroxidase genes, including DypB. To determine whether other bacterial peroxidases participate in the defense against oxidative stress at low $\mathrm{pH}$, we performed a transposon sequencing (Tn-seq) screen ${ }^{19,20}$. To do so, we created a transposon library containing $\sim 100 \mathrm{~K}$ individual transposon mutants and exposed this library to $\mathrm{H}_{2} \mathrm{O}_{2}$ at $\mathrm{pH} 4.5$ for three days, at which point the surviving bacteria were plated. Transposon gene junctions were amplified and sequenced from the recovered bacteria and the sequencing data were analyzed using TRANSIT ${ }^{21}$ (Table S1). These data revealed that of the 18 putative enzymes encoded by Mtb that could be involved in oxidative defense, including peroxidases, catalases, and superoxide 
dismutases, only 2 genes were required for survival in the culture conditions $-D y p B$ and $K a t G$ (Figure 3A). KatG encodes a catalase-peroxidase that is important for defense against oxidative stress in the host ${ }^{22,23}$. Coincidentally, KatG is also required for activation of isoniazid (INH), a central component of anti-TB therapy. By contrast, DypB did not detectably react with INH (data not shown). Approximately $10 \%$ of TB cases are caused by INH-resistant bacteria, many of which have loss-of-function mutations in KatG24. Importantly, KatG variants that do not activate INH often display a concomitant decrease in peroxidase and catalase activity ${ }^{25-27}$. Both the high proportion of KatG mutant bacteria in the human population and studies of virulence in animals suggest that KatG mutants are still capable of growth in vivo ${ }^{23,28}$. The fact that both DypB and the KatG mutants are important for survival in oxidative stress at low $\mathrm{pH}$ suggests that the DypB nanocompartment may provide redundancy that compensates for a loss of KatG in INH resistant strains of Mtb.

In the Tn-seq data, we observed that mutants involved in lipid or cholesterol metabolism were also attenuated when exposed to $\mathrm{H}_{2} \mathrm{O}_{2}$ at pH 4.5 (Figure 3B). M. tuberculosis biology is highly linked to lipid biology; Mtb granulomas are rich in free fatty acids ${ }^{29}$ and the bacteria utilize lipids as a source of nutrition both during macrophage infection ex vivo and growth in vivo ${ }^{30-32}$. Based on the Tn-seq data, we hypothesized that lipids may mediate the sensitivity of DypB mutants to oxidative stress under acidic conditions. The standard Mtb culture medium, 7H9, contains Bovine Serum Albumin (BSA), which has binding sites for fatty acids and may also serve as a cholesterol shuttle in serum ${ }^{33,34}$. We tested whether $\Delta$ operon mutants were susceptible to acid and oxidative stress in Sauton's broth, a minimal medium that lacks BSA, and found that $\Delta$ operon mutants persisted to the same degree as wild-type bacteria (Figure 3C). To test whether lipids bound to BSA mediate the susceptibility of DypB mutants to acid and oxidative stress, we cultured wildtype and $\Delta$ operon Mtb in media constituted with fatty acid-free BSA and exposed the bacteria to 

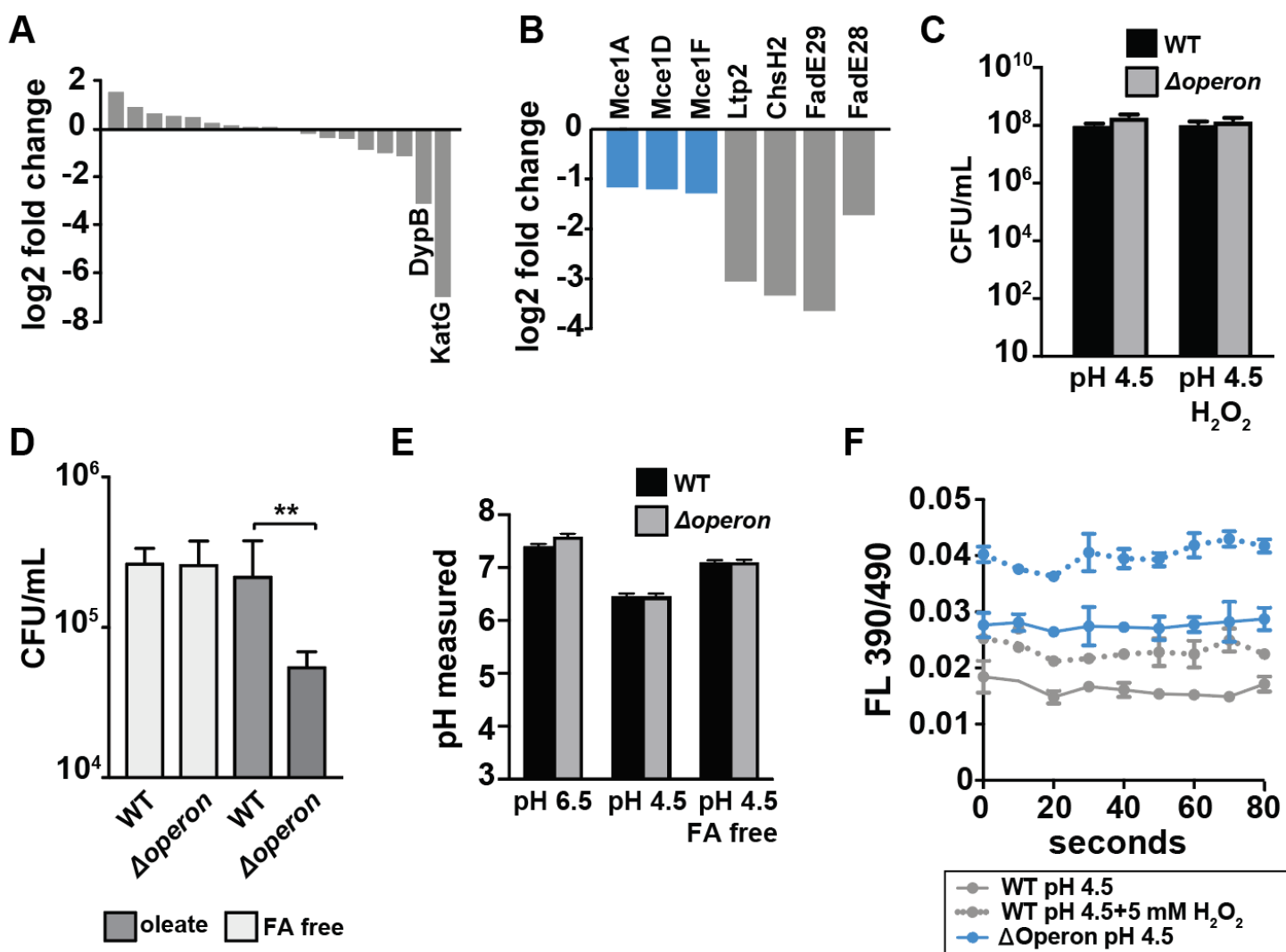

$\mathbf{F}$

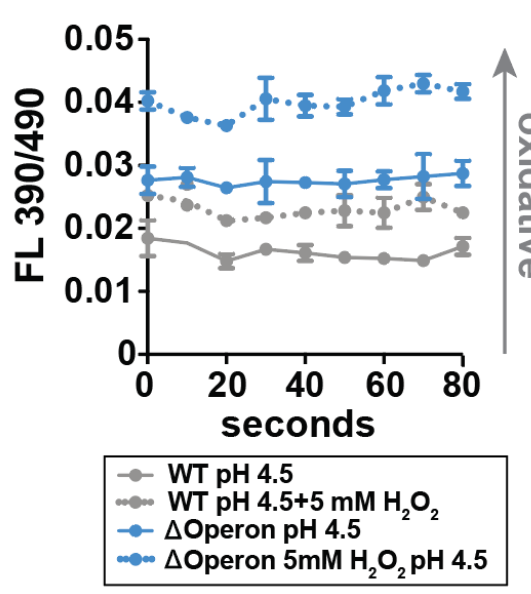

Figure 3. Susceptibility of Mtb nanocompartment mutants to oxidative and acid stress is mediated by free fatty acids. (A) Tn-seq data showing normalized sequence reads per gene for all putative Mtb peroxidases and (B) lipid and cholesterol metabolism Mtb mutants that were significantly attenuated following 72 hour exposure to $2.5 \mathrm{mM} \mathrm{H}_{2} \mathrm{O}_{2}$ at $\mathrm{pH}$ 4.5. (C) CFU enumeration of wild-type Mtb and $\Delta$ operon mutants following 24 hour exposure to 2.5 $\mathrm{mM} \mathrm{H}_{2} \mathrm{O}_{2}$ at $\mathrm{pH} 4.5$ in Sauton's minimal medium and (D) 72 hour exposure to $2.5 \mathrm{mM} \mathrm{H}_{2} \mathrm{O}_{2}$ at $\mathrm{pH} 4.5$ in $7 \mathrm{H} 9$ medium prepared using fatty-acid (FA) free BSA +/- oleic acid (150 UM). (E) Intrabacterial $\mathrm{pH}$ measurements of wild-type and $\Delta$ operon Mtb expressing pUV15-pHGFP following 20 minute exposure to $5 \mathrm{mM} \mathrm{H}_{2} \mathrm{O}_{2}$ at $\mathrm{pH} 6.5$ or $\mathrm{pH} 4.5$. $7 \mathrm{H} 9$ medium was prepared with standard BSA or FA free BSA. (F) Fluorescence emissions of wild-type and $\triangle$ operon Mtb expressing mrx1-roGFP exposed to $5 \mathrm{mM} \mathrm{H}_{2} \mathrm{O}_{2}$ at $\mathrm{pH} 4.5$ in $7 \mathrm{H} 9$ medium for 20 minutes. Data are reported as a ratio of fluorescence emissions following excitation at 490 $\mathrm{nm}$ and $390 \mathrm{~nm}$. Figures are representative of at least 2 (D) or 3 (A-C; E-F) independent experiments. $p$-values were determined using an unpaired t test. ${ }^{*} p<0.05,{ }^{* *} p<0.01$.

$\mathrm{H}_{2} \mathrm{O}_{2}$ at $\mathrm{pH} 4.5$ for three days. In the absence of BSA bound lipids, we found that the $\Delta$ operon mutant was not susceptible to oxidative stress at low pH (Figure 3D). 7H9 medium prepared with lipid free BSA was then reconstituted with oleic acid, an abundant lipid found in mammalian systems $^{35}$. Reconstitution of fatty acid free medium with oleic acid restored toxicity to 
nanocompartment mutants under conditions of acid and oxidative stress (Figure 3D). Taken together, these data suggest that the phagosomal lipids used by Mtb as a carbon source may be toxic to bacteria lacking functional nanocompartments under conditions of oxidative stress

We considered that lipids might disrupt Mtb membranes, resulting in altered $\mathrm{pH}$ homeostasis, and a drop in cytosolic $\mathrm{pH}$. Interestingly, in acidified medium we found that the intracellular $\mathrm{pH}$ of wildtype bacteria dropped from $\sim \mathrm{pH} 7.5$ to $\sim \mathrm{pH} 6.2$ and this decrease was dependent on the presence of albumin-bound fatty acids (Figure 3E). These data confirm published results demonstrating that the cytosolic $\mathrm{pH}$ of Mtb drops significantly during acid exposure. ${ }^{18} \mathrm{~A}$ lower cytosolic $\mathrm{pH}$ would possibly impair the function of many other bacterial enzymes important for resisting oxidative stress and place increasing importance on enzymes that can function in acidic environments, such as DypB. ${ }^{23}$ Taken together, these data may explain why functional nanocompartments are critical for bacterial survival when Mtb is exposed to acid and oxidative stress in a fatty acid-rich environment.

We next sought to test whether nanocompartment mutants have altered redox homeostasis in the presence of oxidative stress. We transformed wild-type and mutant strains with Mrx1-roGFP, a fluorescent reporter of redox potential in mycobacteria ${ }^{36}$. Remarkably, the $\Delta$ operon mutant had an intracellular environment at baseline that was more oxidizing than wild-type bacteria exposed to $\mathrm{H}_{2} \mathrm{O}_{2}$ (Figure 3F), indicating a failure of redox homeostasis in mutants lacking the DypB encapsulin system. This oxidizing cellular environment was further enhanced by treatment with $\mathrm{H}_{2} \mathrm{O}_{2}$ (Figure 3F). Thus, mutants lacking DypB-containing nanocompartments exhibit significant dysregulation of redox homeostasis. 

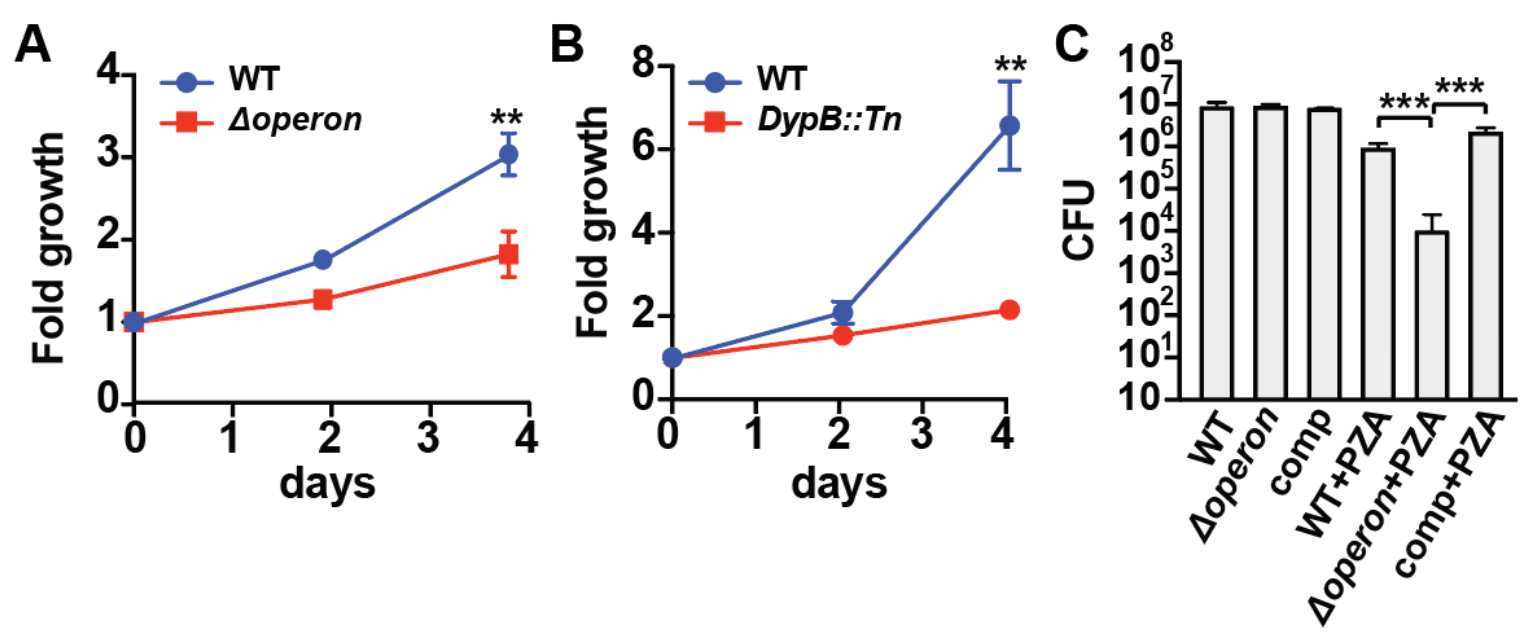

Figure 4. Nanocompartment mutants are attenuated for survival in macrophages and are more susceptible to pyrazinamide treatment. CFU enumeration of wild-type Mtb and (A) $\Delta$ operon or (B) DypB::Tn mutants during infection of murine bone marrow-derived macrophages. Macrophages were infected with a bacterial MOI of 1 and CFUs were enumerated immediately following phagocytosis and at days 2 and 4 . Error bars are SD from 4 replicate wells. (C) CFU enumeration of wild-type and Mtb $\Delta$ operon mutants following 72 hour exposure to pyrazinamide $(24 \mu \mathrm{g} / \mathrm{mL})$ and $\mathrm{H}_{2} \mathrm{O}_{2}(2.5 \mathrm{mM})$ in acidified $7 \mathrm{H} 9$ medium $(\mathrm{pH}$ 5.5). Comp=Loperon $+p$ Operon. Figures are representative of at least 2 independent experiments; $p$-values were determined using an unpaired t test. ${ }^{* *} p<0.01 ;{ }^{* * *} p<0.001$.

Our in vitro findings that DypB nanocompartments are important for defense against a combination of oxidative stress, low $\mathrm{pH}$, and fatty acids suggested that this system may be important for Mtb survival in the phagosomal environment. Therefore, we sought to determine whether DypB nanocompartments are required for bacterial growth in host cells. We found that both the $D y p B:: T n$ and $\Delta$ operon mutants had impaired growth in murine bone-marrow derived macrophages that was manifest by two days after infection (Figure 4A, 4B). One of the antibiotics used to treat TB infection, Pyrazinamide (PZA), is most effective in low pH environments, such as the phagolysosome. PZA is thought to disrupt the cell wall of Mtb, which leads to acidification of the bacterial cytosol. A recent study in human TB patients demonstrated that the $\mathrm{pH}$ of necrotic lung cavities is $\sim 5.5$, a finding that may also explain the efficacy of pyrazinamide in treating Mtb infection ${ }^{37}$. Since DypB is critical for protection of Mtb against oxidative stress at low $\mathrm{pH}$, we considered whether nanocompartments may mediate Mtb resistance to PZA. To test bacterial 
susceptibility to PZA, we treated wild-type and $\triangle$ operon Mtb with PZA in the presence of $\mathrm{H}_{2} \mathrm{O}_{2}$ at $\mathrm{pH}$ 5.5. We found that mutants lacking the DypB nanocompartment were sensitive to concentrations of PZA that did not impact growth of the wild-type bacteria (Figure 4C).

Encapsulin nanocompartments are a largely uncharacterized proteinaceous organelle widespread in bacteria and archaea. Here we provide some of the first functional data demonstrating the physiological significance of a nanocompartment and its cargo in any organism. Furthermore, our finding that encapsulation of DypB promotes its function in resisting oxidative stress represents the first demonstration that encapsulation can promote the function of an encapsulated enzyme in an endogenous setting. Furthermore, we have demonstrated that an encapsulin system is important for resisting oxidative stress in the context of a globally significant pathogen, Mycobacterium tuberculosis, expanding our understanding of how this pathogen combats the hostile in vivo environment. 


\section{Materials and Methods}

Ethics statement. All procedures involving the use of mice were approved by the University of California, Berkeley Institutional Animal Care and Use Committee (protocol no. R353-1113B). All protocols conform to federal regulations, the National Research Council's Guide for the Care and Use of Laboratory Animals, and the Public Health Service's Policy on Humane Care and Use of Laboratory Animals.

Mycobacterium tuberculosis bacterial strains and plasmids. The $M$. tuberculosis strain H37Rv was used for all experiments. The transposon mutants DypB::Tn and Rv1762c::Tn were picked from an arrayed transposon mutant library generated at the Broad Institute. The $\Delta$ Operon, $\Delta \mathrm{Cfp} 29$, and $\Delta \mathrm{DypB}$ strains were made by homologous recombination using the pMSG361 vector ${ }^{38}$. For genetic complementation studies, the region encoding GFP and KanR in pUV15tetORm ${ }^{39}$ was substituted via GoldenGate cloning with open reading frames for Rv0798c, Rv0799c, or the whole nanocompartment operon (Rv0798-99c). Expression of the complementation constructs was induced with anhydrotetracycline (200 ng/mL). To measure redox homeostasis, strains were transformed with pMV762-mrx1-roGFP2 ${ }^{36}$. To measure intrabacterial $\mathrm{pH}$, strains were transformed with pUV15-pHGFP (Addgene). The transposon mutant library for Tn-Seq was generated in M. tuberculosis using the ФMycoMarT7 transposon donor plasmid.

M. tuberculosis bacterial cell culture. For infections, M. tuberculosis was grown to mid-log phase $\left(\mathrm{OD}_{600}=0.5-1.0\right)$ in Middlebrook $7 \mathrm{H} 9$ liquid medium supplemented with $10 \%$ albumindextrose-saline, $0.4 \%$ glycerol, and $0.05 \%$ Tween- 80 or on solid $7 \mathrm{H} 10$ agar plates supplemented with Middlebrook OADC (BD Biosciences) and 0.4\% glycerol. When specified, 
Tween-80 was substituted with $0.05 \%$ Tyloxapol, and $10 \%$ albumin-dextrose-saline was prepared with fatty acid free BSA (Sigma-Aldrich). Sauton's media was prepared with tyloxapol as previously specified 40 .

DypB activity assays. Activity of the encapsulated and unencapsulated DypB was performed using methods adapted from Contreras et al. 2014. Briefly, DypB concentration for the encapsulated and unencapsulated DypB was determined by absorbance of the heme prosthetic group at $411 \mathrm{~nm}$. Reactions were performed using $5 \mathrm{nM}$ DypB, $480 \mathrm{nM} \mathrm{H}_{2} \mathrm{O}_{2}$, and $480 \mathrm{nM}$ 2,2'azino-bis (3-ethylbenzothiazoline-6-sulfonic acid) (ABTS) in 100mM sodium citrate buffer pH 46. Product formation was monitored over 20 minutes via absorbance at $420 \mathrm{~nm}$ using a Varian Cary® 50 UV-Vis Spectrophotometer (Agilent).

Nanocompartment Purification from Mtb. For each purification, $1.5 \mathrm{~L}$ of $M$. tuberculosis was grown to mid-log phase in standard 7H9 and washed with PBS. Bacteria were pelleted and lysed in buffer by bead beating (for $50 \mathrm{~mL}$ of buffer, PBS with $1 \mathrm{mM}$ PMSF was supplemented with 50 mg lysozyme, $20 \mathrm{U}$ DNasel, and $100 \mu \mathrm{g}$ RNase A). Lysates were passaged twice through $0.2 \mu \mathrm{m}$ filters before removal from the BSL3. Clarified lysates were prepared by centrifugation at $20,000 \times g$ for 20 minutes in a JA-20 rotor. Following clarification, lysates were layered onto top of a $38 \%$ sucrose cushion and centrifuged for 18 hours at $100,000 \times g$ in a type 50.2 Ti rotor. The supernatant was discarded and the pellet was resuspended in $200 \mu \mathrm{L}$ of PBS. Resuspended pellets were layered on top of a 10-50\% sucrose gradient and centrifuged for 21 hours at $100,000 \times \mathrm{g}$ in a SW 41 Ti rotor. The gradient was fractionated and aliquots from each fraction were analyzed by SDS-PAGE for the presence of Cfp29. 
Expression of Holo-nanocompartment and naked DypB in E. coli. Plasmids for the expression of the Holo-nanocompartment (DypB-loaded) and naked DypB constructs were designing using Gibson Assembly (NEB). Each construct was cloned into a pET-14-based destination vector containing a T7 promoter. The naked DypB construct contained an N-terminal poly-histidine tag for affinity purification. These constructs were transformed into E. coli BL21 (DE3) LOBSTR cells for protein overexpression. Cells were grown in LB media containing 60 $\mu \mathrm{g} / \mathrm{mL}$ kanamycin at $37^{\circ} \mathrm{C}$ with shaking at 250rpm until cultures reached an optical density $\left(\mathrm{OD}_{600}=0.5-0.6\right)$. Samples were then induced with $0.5 \mathrm{mM} \mathrm{IPTG}$ and grown overnight at $18^{\circ} \mathrm{C}$. Liquid cultures were harvested by centrifugation at $5000 \times g$ for 20 minutes at $4^{\circ} \mathrm{C}$, flash frozen in liquid nitrogen, and then stored at $-80^{\circ} \mathrm{C}$ for future use.

Purification of Holo-nanocompartment complex from E. coli. Cell pellets ( $5 \mathrm{~g}$ dry cell mass) were thawed at room temperature and resuspended in $50 \mathrm{~mL}$ of lysis buffer $(20 \mathrm{mM}$ Tris- $\mathrm{HCl} \mathrm{pH}$ 8, $150 \mathrm{mM} \mathrm{NH}_{4} \mathrm{Cl}, 20 \mathrm{mM} \mathrm{MgCl}_{2}$ ) supplemented with $50 \mathrm{mg}$ lysozyme, $20 \mathrm{U}$ DNasel, $100 \mathrm{ug}$ RNase A. Samples were lysed by three passages through an Avestin EmulsiFlex-C3 homogenizer and clarified via centrifugation $\left(15,000 \times \mathrm{g}, 30 \mathrm{~min}, 4^{\circ} \mathrm{C}\right)$. The clarified lysate was then spun at $110,000 \times g$ for 3 hours at $4^{\circ} \mathrm{C}$. The supernatant was discarded and the resulting pellet was resuspended with wash buffer $\left(20 \mathrm{mM}\right.$ Tris $\mathrm{pH} 8,150 \mathrm{mM} \mathrm{NH}_{4} \mathrm{Cl}, 20 \mathrm{mM} \mathrm{MgCl} 2$ supplemented with 1 X Cell Lytic B (Sigma-Aldrich). The sample was then spun at 4,000 $\mathrm{g} g$ at $4^{\circ} \mathrm{C}$ for $10 \mathrm{~min}$ followed by removing the supernatant and resuspension of the pellet in $4 \mathrm{~mL}$ of $50 \mathrm{mM}$ Tris- $\mathrm{HCl} \mathrm{pH} \mathrm{8,} 300 \mathrm{mM} \mathrm{NaCl}$. The sample was then incubated at room temperature for 10 minutes to allow for solubilization and then centrifuged at $4000 \times g$ at $4^{\circ} \mathrm{C}$ for 10 minutes to remove insoluble material. The resulting supernatant was then concentrated using Vivaspin® 6 100,000 MWCO concentrator columns (Sartorius). The sample was then purified via size exclusion chromatography using a Superose ${ }^{\mathrm{TM}} 6$ Increase column (GE Life Sciences) and 
fractions were analyzed by SDS-PAGE using 4-20\% Criterion ${ }^{\mathrm{TM}}$ polyacrylamide gels (Bio-Rad) and visualized with GelCode Blue stain (ThermoFisher).

Purification of unencapsulated DypB from E. coli. Cell pellets ( $5 \mathrm{~g}$ dry cell mass) were thawed at room temperature and resuspended in $50 \mathrm{~mL}$ of buffer $\mathrm{A}(25 \mathrm{mM}$ Tris $\mathrm{HCl} \mathrm{pH} 7.5$, $150 \mathrm{mM} \mathrm{NaCl}, 20 \mathrm{mM}$ imidazole) supplemented with 50 mg lysozyme, $20 \mathrm{U}$ DNasel, $100 \mu \mathrm{g}$ RNase A. Samples were lysed by three passages through an Avestin EmulsiFlex-C3 homogenizer and clarified via centrifugation $\left(15,000 \times \mathrm{g}, 30 \mathrm{~min}, 4^{\circ} \mathrm{C}\right)$. The resulting supernatant was then bound to HisPur ${ }^{\mathrm{TM}}$ Ni-NTA resin (ThermoFisher Scientific) for 90 minutes at $4^{\circ} \mathrm{C}$ and then applied to a gravity column. The nickel resin was then washed with 30 resin volumes of buffer $\mathrm{B}(25 \mathrm{mM}$ Tris- $\mathrm{HCl} \mathrm{pH} \mathrm{7.5,} 150 \mathrm{mM} \mathrm{NaCl}, 40 \mathrm{mM}$ imidazole) prior to eluting with buffer $\mathrm{C}$ (25 mM Tris- $\mathrm{HCl} \mathrm{pH} 7.5,150 \mathrm{mM} \mathrm{NaCl}, 350 \mathrm{mM}$ imidazole). The eluate was then concentrated using Vivaspin® 20 10,000 MWCO concentrator columns (Sartorius) and desalted into 25mM Tris pH 8, 300mM NaCl using Econo-Pac®10DG desalting columns (BioRad). The SUMO tag was removed upon addition of SUMO protease at a 1: 300 (SUMO protease: DypB) molar ratio and incubating overnight at $4^{\circ} \mathrm{C}$. Purification was finished by size exclusion chromatography with a Superose ${ }^{\mathrm{TM}} 6$ Increase column (GE Life Sciences).

Negative stain Transmission Electron Microscopy. Nanocompartment samples were diluted to $50 \mathrm{nM}$ and applied to Formvar/ carbon-coated copper grids. The grids were then washed with MilliQ water three times followed by staining with $2 \%(w / v)$ uranyl acetate. Grids were examined using the FEI Tecnai 12, 120kV transmission electron microscope, and images were captured with a charge-coupled device (CCD) camera. 
Cathepsin B proteolysis assay. Proteolysis was performed using $2 \mu \mathrm{M}$ of encapsulated or unencapsulated DypB in proteolysis buffer (100mM Sodium acetate $\mathrm{pH} 5,2 \mathrm{mM}$ DTT) with or without the addition of $1 \mu \mathrm{M}$ Cathepsin B (Sigma-Aldrich Cat\# 219362). Reactions were run for 14 hours at $40^{\circ} \mathrm{C}$ followed by analysis of samples by SDS-PAGE using $4-20 \%$ Criterion $^{\mathrm{TM}}$ polyacrylamide gels (Bio-Rad) and visualized with GelCode Blue stain (ThermoFisher).

Exposure to oxidative and pH stress. M. tuberculosis was grown to mid-log phase in 7H9 media. Bacteria were diluted to $\mathrm{OD}_{600}=0.1$ in $10 \mathrm{~mL}$ of specified media at $\mathrm{pH} 4.5-6.5$ and $\mathrm{H}_{2} \mathrm{O}_{2}$ was added to bacterial cultures at specified concentrations. Bacteria were incubated with stressors for 24 or 72 hours. CFUs were enumerated by diluting bacteria in PBS with $0.05 \%$ Tween-80 and plating serial dilutions on $7 \mathrm{H} 10$ agar.

Measurement of redox homeostasis. M. tuberculosis strains were transformed with a plasmid expressing mrx1-roGFP2 and grown to mid-log phase in 7H9. Bacteria were diluted to $\mathrm{OD}_{600}=$ 0.25 in $200 \mu \mathrm{L}$ of specified media and added to 96 well plates. Upon addition of $\mathrm{H}_{2} \mathrm{O}_{2}(5 \mathrm{mM})$, fluorescent emissions were recorded at $510 \mathrm{~nm}$ after excitation at $390 \mathrm{~nm}$ and $490 \mathrm{~nm}$ using a Spectramax M3 spectrophotometer. Values reported are emissions ratios $(390 \mathrm{~nm} / 490 \mathrm{~nm})$ and were measured 20 minutes following addition of $\mathrm{H}_{2} \mathrm{O}_{2}$.

Measurement of intrabacterial pH. M. tuberculosis strains were transformed with a plasmid expressing pHGFP and grown to mid-log phase in 7 H9. To prepare standards, $1.5 \times 10^{\wedge} 8$ bacterial cells were pelleted and resuspended in $400 \mu \mathrm{L}$ lysis buffer $(50 \mathrm{mM}$ Tris- $\mathrm{HCl} \mathrm{pH}$ 7.5, 5 mM EDTA, 0.6\% SDS, 1 mM PMSF) before bead beating. Cell debris were pelleted and clarified lysates were kept at $4^{\circ} \mathrm{C}$ until use, at which point $10 \mu \mathrm{L}$ of clarified lysate were added to $200 \mu \mathrm{L}$ of medium with varying $\mathrm{pH}$ levels (4.5-8.0). To prepare samples, $1.5 \times 10^{\wedge} 8$ bacterial 
cells were pelleted and washed with PBS twice before being resuspended in specified media and diluted to $\mathrm{OD}_{600}=0.5$ in $200 \mu \mathrm{L}$ of medium and added to 96 wells plates. Upon addition of $\mathrm{H}_{2} \mathrm{O}_{2}(5 \mathrm{mM})$, fluorescent emissions were recorded at $510 \mathrm{~nm}$ following excitation at $395 \mathrm{~nm}$ and $475 \mathrm{~nm}$. Values reported were interpolated from 395/475 ratios obtained from the standard curve.

Western blot analysis of Cfp29 expression. M. tuberculosis strains were grown to mid-log phase in 7H9 medium. Bacteria were pelleted and washed twice with PBS prior to resuspension in lysis buffer (50 mM Tris-HCl pH 7.5, 5 mM EDTA, 0.6\% SDS, 1 mM PMSF). Samples were lysed using a bead-beater, and cell debris were pelleted. Clarified lysates were heat-sterilized at $100^{\circ} \mathrm{C}$ for 15 minutes and frozen prior to use. Total protein lysates were analyzed by SDSPAGE using precast Tris-HCl 4-20\% criterion gels (Bio-Rad). Primary polyclonal antibodies for Cfp29 were generated by GenScript USA Inc via immunization of rabbits with three peptides from the protein sequence. HRP-conjugated goat anti-rabbit IgG secondary antibodies were used (sc-2030; Santa Cruz Biotechnology). Western Lightning Plus-ECL chemiluminescence substrate (Perkin Elmer) was used and blots were developed using a ChemiDoc MP System (Bio-Rad).

Infection of murine macrophages. Macrophages were derived from bone marrow of C57BL/6 mice by flushing cells from femurs. Cells were cultured in DMEM supplemented with $10 \%$ FBS and $10 \%$ supernatant from 3T3-M-CSF cells for 6 days, with feeding on day 3. After differentiation, BMDMs continued to be cultured in BMDM media containing M-CSF. For infection, BMDMs were seeded at a density of $5 \times 10^{\wedge} 4$ cells per well in a 96-well dish. BMDMs were allowed to adhere overnight and then infected with DMEM supplemented with $5 \%$ FBS and $5 \%$ horse serum (BMDMs) at a multiplicity of infection of 1 . Following a 4-hour phagocytosis 
period, infection medium was removed and cells were washed with room temperature PBS before fresh, complete medium was added. For CFU enumeration, medium was removed and cells were lysed in water with $0.5 \%$ Triton- $X$ and incubated at $37^{\circ} \mathrm{C}$ for 10 minutes. Following the incubation, lysed cells were resuspended and serially diluted in PBS with $0.05 \%$ Tween-80. Dilutions were plated on $7 \mathrm{H} 10$ plates.

Transposon-sequencing screen. A transposon mutant library in H37Rv was grown to mid-log phase in 7H9. Bacteria were diluted to $\mathrm{OD}_{600}=0.1$ in $10 \mathrm{~mL} 7 \mathrm{H} 9$ at $\mathrm{pH} 4.5$ with $2.5 \mathrm{mM} \mathrm{H}_{2} \mathrm{O}_{2}$. Mutants were exposed to these stressors for 72 hours and then diluted to $15,000 \mathrm{CFU} / \mathrm{mL}$ in PBS with $0.05 \%$ Tween-80. Approximately 30 thousand bacteria were plated onto six $245 \mathrm{~mm} \mathrm{x}$ $245 \mathrm{~mm}$ 7H10 plates supplemented with $0.05 \%$ Tween-80 and Kanamycin $(50 \mu \mathrm{g} / \mathrm{mL})$. Control libraries were not exposed to low $\mathrm{pH}$ or $\mathrm{H}_{2} \mathrm{O}_{2}$ and were plated onto $7 \mathrm{H} 10$ plates. Colonies grew for 21 days and were collected for genomic DNA isolation. Samples for sequencing were prepared by the University of California, Davis Genome Center DNA Technologies Core by following the protocol outlined by Long et $\mathrm{al}^{41}$. PE100 reads were run on an Illumina HiSeq with 20 million reads per sample. Sample alignment and TRANSIT pre-processing were performed by the University of California, Davis Bioinformatics Group as previously outlined ${ }^{21}$. TRANSIT analysis was performed as specified by DeJesus et al ${ }^{21}$. Resampling analysis was preformed using the reference genome H37RvBD_prot and the following parameters: for global options, $0 \%$ of the $\mathrm{N}$ - and $\mathrm{C}$ - terminus were ignored; for resampling options, 10,000 samples were taken and normalized using the TTR function. Correction for genome positional bias was performed. Statistical significance was determined by $p$ value $\leq 0.05$ and $\log 2$ fold change $\leq-1$ or by $p$ adjusted value $\leq 0.05$ 


\section{Bibliography}

1. Nichols, R. J., Cassidy-Amstutz, C., Chaijarasphong, T. \& Savage, D. F. Encapsulins: molecular biology of the shell. Crit Rev Biochem Mol Biol 52, 583-594 (2017).

2. Giessen, T. W. \& Silver, P. A. Widespread distribution of encapsulin nanocompartments reveals functional diversity. Nat. Microbiol. 2, 17029 (2017).

3. Kerfeld, C. A., Aussignargues, C., Zarzycki, J., Cai, F. \& Sutter, M. Bacterial microcompartments. Nat. Rev. Microbiol. 16, 277-290 (2018).

4. Uebe, R. \& Schüler, D. Magnetosome biogenesis in magnetotactic bacteria. Nat. Rev. Microbiol. 14, 621-637 (2016).

5. Ferousi, C. et al. Iron assimilation and utilization in anaerobic ammonium oxidizing bacteria. Curr. Opin. Chem. Biol. 37, 129-136 (2017).

6. Giessen, T. W. et al. Large protein organelles form a new iron sequestration system with high storage capacity. Elife 8, (2019).

7. Nichols, R. J. et al. Discovery and characterization of a novel family of prokaryotic nanocompartments involved in sulfur metabolism. bioRxiv (2020). at $<$ https://doi.org/10.1101/2020.05.24.113720>

8. McHugh, C. A. et al. A virus capsid-like nanocompartment that stores iron and protects bacteria from oxidative stress. EMBO J. 33, 1896-1911 (2014).

9. Contreras, $\mathrm{H}$. et al. Characterization of a Mycobacterium tuberculosis nanocompartment and its potential cargo proteins. J. Biol. Chem. 289, 18279-18289 (2014).

10. Zhang, Y. J. et al. Tryptophan biosynthesis protects mycobacteria from CD4 T-cellmediated killing. Cell 155, 1296-1308 (2013).

11. Weldingh, K. \& Andersen, P. Immunological evaluation of novel Mycobacterium tuberculosis culture filtrate proteins. FEMS Immunol. Med. Microbiol. 23, 159-164 (1999).

12. Rosenkrands, I. et al. Identification and characterization of a 29-kilodalton protein from Mycobacterium tuberculosis culture filtrate recognized by mouse memory effector cells. Infect. Immun. 66, 2728-2735 (1998).

13. Cassidy-Amstutz, C. et al. Identification of a minimal peptide tag for in vivo and in vitro loading of encapsulin. Biochemistry 55, 3461-3468 (2016).

14. Uchida, T., Sasaki, M., Tanaka, Y. \& Ishimori, K. A Dye-Decolorizing Peroxidase from Vibrio cholerae. Biochemistry 54, 6610-6621 (2015).

15. Ahmad, M. et al. Identification of DypB from Rhodococcus jostii RHA1 as a lignin peroxidase. Biochemistry 50, 5096-5107 (2011).

16. Mishra, S. \& Imlay, J. Why do bacteria use so many enzymes to scavenge hydrogen peroxide? Arch. Biochem. Biophys. 525, 145-160 (2012).

17. El-Benna, J., Dang, P. M.-C., Gougerot-Pocidalo, M. A., Marie, J. C. \& Braut-Boucher, F. p47phox, the phagocyte NADPH oxidase/NOX2 organizer: structure, phosphorylation and implication in diseases. Exp Mol Med 41, 217-225 (2009).

18. Vandal, O. H., Pierini, L. M., Schnappinger, D., Nathan, C. F. \& Ehrt, S. A membrane protein preserves intrabacterial $\mathrm{pH}$ in intraphagosomal Mycobacterium tuberculosis. Nat. Med. 14, 849-854 (2008).

19. van Opijnen, T. \& Camilli, A. Transposon insertion sequencing: a new tool for systems-level analysis of microorganisms. Nat. Rev. Microbiol. 11, 435-442 (2013).

20. Griffin, J. E. et al. Cholesterol catabolism by Mycobacterium tuberculosis requires transcriptional and metabolic adaptations. Chem. Biol. 19, 218-227 (2012).

21. DeJesus, M. A., Ambadipudi, C., Baker, R., Sassetti, C. \& loerger, T. R. TRANSIT--A Software Tool for Himar1 TnSeq Analysis. PLoS Comput. Biol. 11, e1004401 (2015). 
22. Li, Z., Kelley, C., Collins, F., Rouse, D. \& Morris, S. Expression of katG in Mycobacterium tuberculosis is associated with its growth and persistence in mice and guinea pigs. J. Infect. Dis. 177, 1030-1035 (1998).

23. Pym, A. S., Saint-Joanis, B. \& Cole, S. T. Effect of katG mutations on the virulence of Mycobacterium tuberculosis and the implication for transmission in humans. Infect. Immun. 70, 4955-4960 (2002).

24. Seifert, M., Catanzaro, D., Catanzaro, A. \& Rodwell, T. C. Genetic mutations associated with isoniazid resistance in Mycobacterium tuberculosis: a systematic review. PLoS One 10, e0119628 (2015).

25. Wei, C.-J., Lei, B., Musser, J. M. \& Tu, S.-C. Isoniazid activation defects in recombinant Mycobacterium tuberculosis catalase-peroxidase (KatG) mutants evident in InhA inhibitor production. Antimicrob. Agents Chemother. 47, 670-675 (2003).

26. Mo, L. et al. Three-dimensional model and molecular mechanism of Mycobacterium tuberculosis catalase-peroxidase (KatG) and isoniazid-resistant KatG mutants. Microb Drug Resist 10, 269-279 (2004).

27. DeVito, J. A. \& Morris, S. Exploring the structure and function of the mycobacterial KatG protein using trans-dominant mutants. Antimicrob. Agents Chemother. 47, 188-195 (2003).

28. Nieto R, L. M. et al. Virulence of Mycobacterium tuberculosis after Acquisition of Isoniazid Resistance: Individual Nature of katG Mutants and the Possible Role of AhpC. PLoS One 11, e0166807 (2016).

29. Kim, M.-J. et al. Caseation of human tuberculosis granulomas correlates with elevated host lipid metabolism. EMBO Mol. Med. 2, 258-274 (2010).

30. Marrero, J., Rhee, K. Y., Schnappinger, D., Pethe, K. \& Ehrt, S. Gluconeogenic carbon flow of tricarboxylic acid cycle intermediates is critical for Mycobacterium tuberculosis to establish and maintain infection. Proc. Natl. Acad. Sci. USA 107, 9819-9824 (2010).

31. Russell, D. G., Huang, L. \& VanderVen, B. C. Immunometabolism at the interface between macrophages and pathogens. Nat. Rev. Immunol. 19, 291-304 (2019).

32. Russell, D. G. Mycobacterium tuberculosis and the intimate discourse of a chronic infection. Immunol. Rev. 240, 252-268 (2011).

33. van der Vusse, G. J. Albumin as fatty acid transporter. Drug Metab Pharmacokinet 24, 300-307 (2009).

34. Sankaranarayanan, S. et al. Serum albumin acts as a shuttle to enhance cholesterol efflux from cells. J. Lipid Res. 54, 671-676 (2013).

35. The PLOS ONE Staff. Correction: comprehensive profiling of plasma fatty acid concentrations in young healthy canadian adults. PLoS One 10, e0128167 (2015).

36. Bhaskar, A. et al. Reengineering redox sensitive GFP to measure mycothiol redox potential of Mycobacterium tuberculosis during infection. PLoS Pathog. 10, e1003902 (2014).

37. Kempker, R. R. et al. Lung Tissue Concentrations of Pyrazinamide among Patients with Drug-Resistant Pulmonary Tuberculosis. Antimicrob. Agents Chemother. 61, (2017).

38. Rosenberg, O. S. et al. Substrates Control Multimerization and Activation of the MultiDomain ATPase Motor of Type VII Secretion. Cell 161, 501-512 (2015).

39. Ehrt, S. et al. Controlling gene expression in mycobacteria with anhydrotetracycline and Tet repressor. Nucleic Acids Res. 33, e21 (2005).

40. Larsen, M. H., Biermann, K. \& Jacobs, W. R. in Current Protocols in Microbiology 6, (John Wiley \& Sons, Inc., 2007).

41. Long, J. E. et al. Identifying essential genes in Mycobacterium tuberculosis by global phenotypic profiling. Methods Mol. Biol. 1279, 79-95 (2015). 


\section{Acknowledgments.}

We thank Tom loerger and Michael DeJesus for assistance with TRANSIT analysis and Amit Singh for the kind gift of pMV762-mrx1-roGFP2. We thank Jeff Cox and members of the Cox lab for helpful discussions. This work was supported by funding from the Center for Emerging and Neglected disease for funding to KAL, the National Institute of General Medical Sciences (R01GM129241) to DFS and the National Institute of Allergy and Infectious Diseases (1R01Al143722) to SAS. 

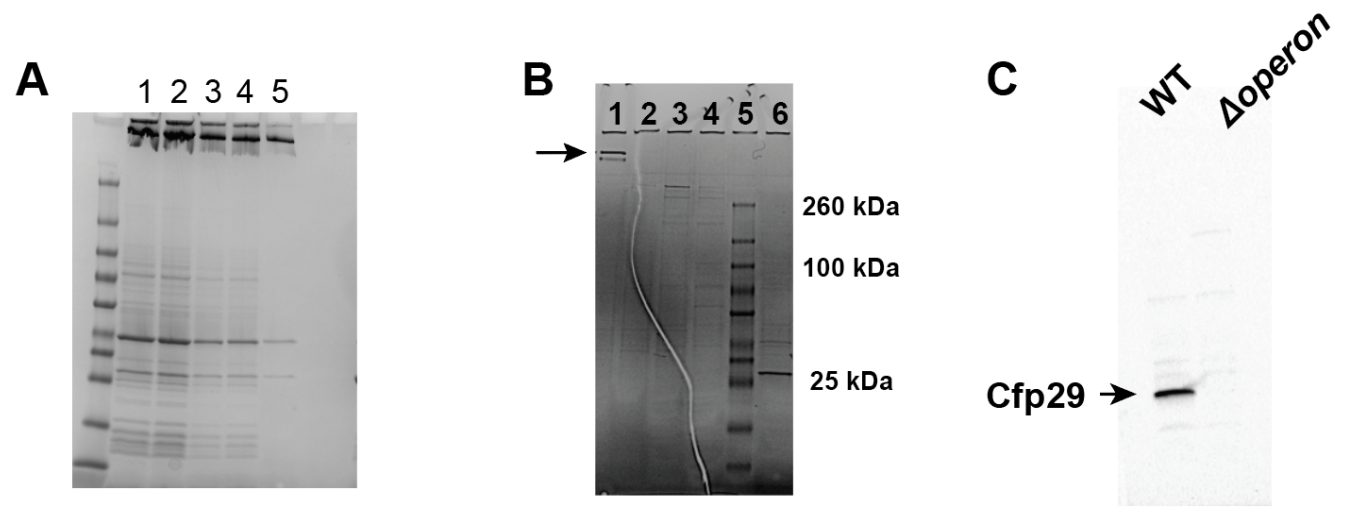

D

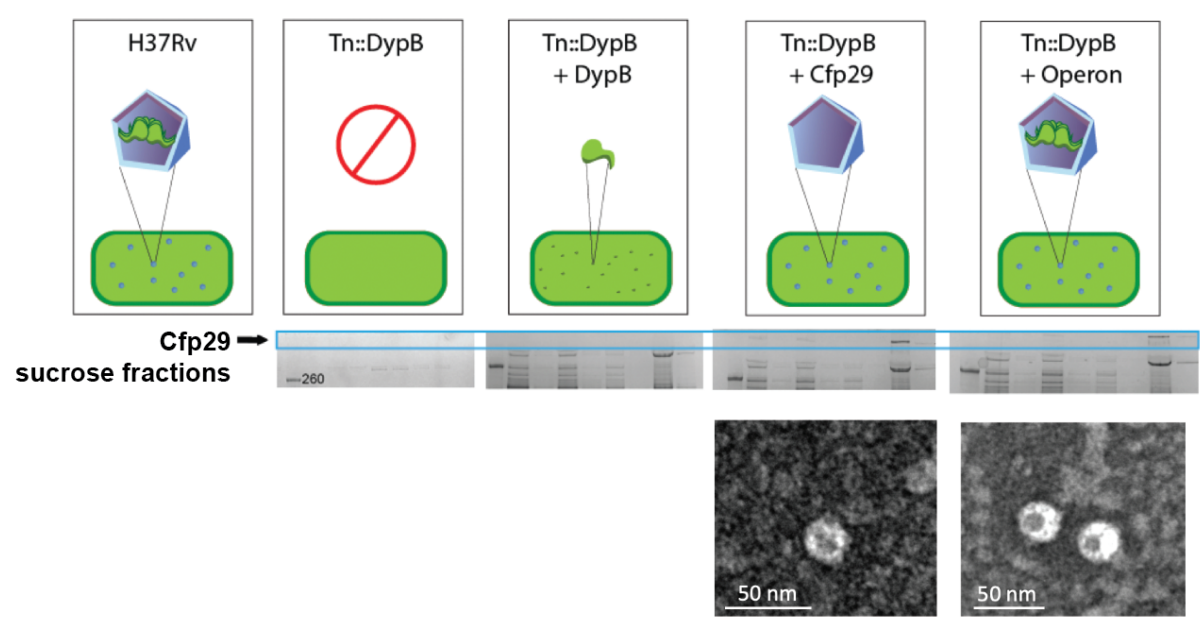

Figure S1. (A) Coomassie stained SDS-PAGE of fractions collected during purification of nanocompartments heterologously expressed in E. coli: (1) Ultracentrifugation pellet post CellLytic B solubilization (2) Size exclusion chromatography input (3) lane 1 diluted (4) lane 2 diluted (5) encapsulin fraction from size exclusion. (B) Coomassie stained SDS-PAGE of sucrose fractions collected during purification of nanocompartments from wild-type Mtb lysates: (1) Fraction containing assembled encapsulin nanocompartment complexes (5) Ladder (6) Lane 1 boiled in SDS for 30 minutes to dissociate encapsulin nanocompartment into monomers. (C) Western blot for Cfp29 from wild-type Mtb (lane 1) and $\Delta$ operon mutant (lane 2) lysates. (D) Complementation strategy schematic for $D y p B:: T n$ mutants (top). DypB::Tn mutants were transformed with ATc-inducible complementation constructs encoding the unencapsulated cargo

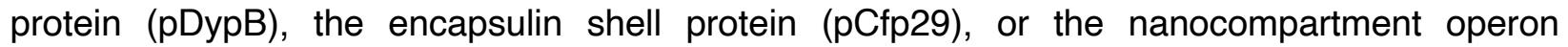
(pOperon). Lysates from each strain were used for nanocompartment purification. Sucrose fractions containing high molecular weight Cfp29 protomers were identified in complemented strains expressing the encapsulin shell and the operon (middle) and were analyzed using TEM (bottom). 


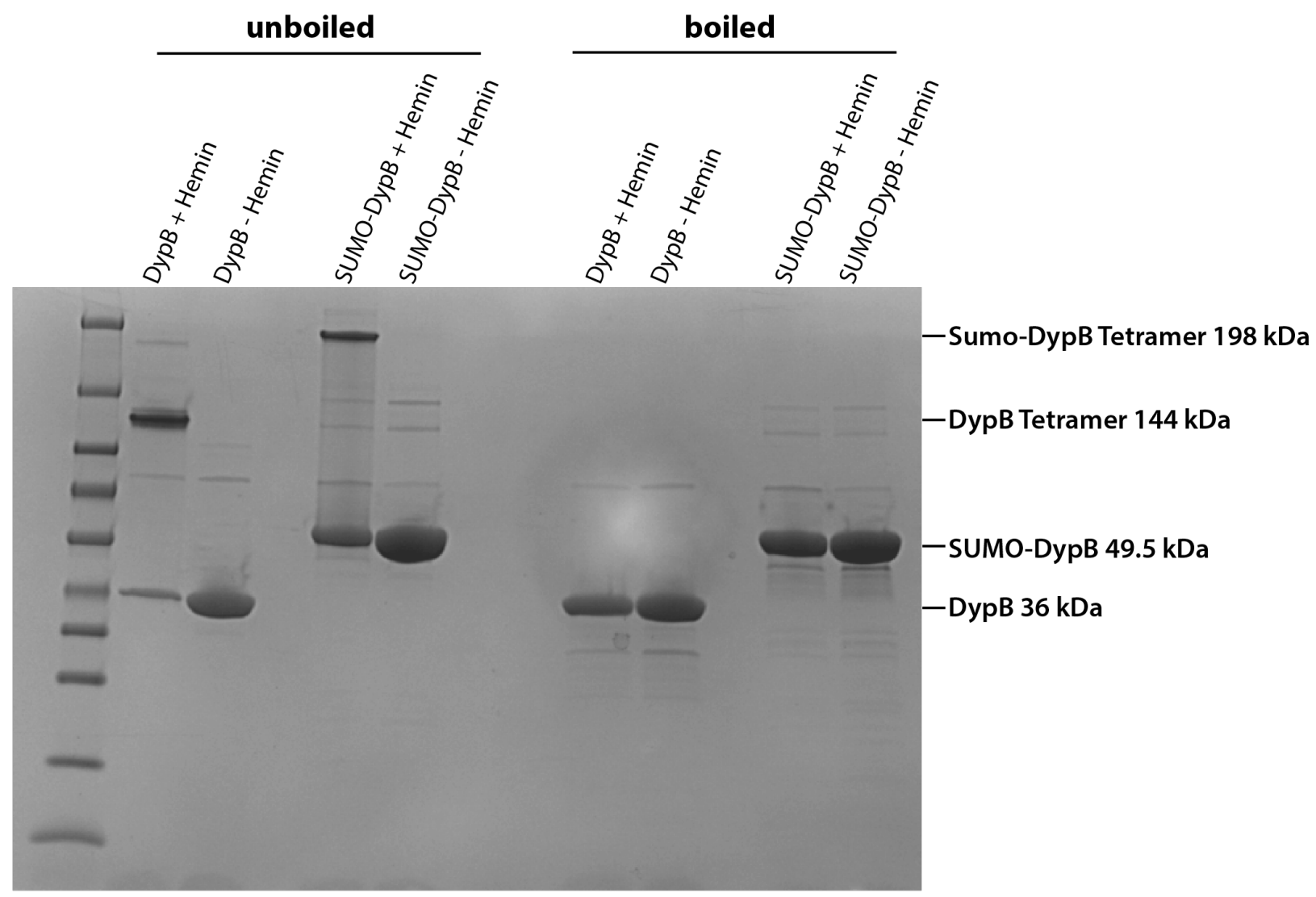

Figure S2. SDS-PAGE analysis of DypB purified from E. coli. DypB samples with or without addition of hemin were analyzed by SDS-PAGE. Samples were loaded either in their unboiled native state (left half) or heat-denatured by boiling at $95^{\circ} \mathrm{C}$ for 15 minutes. Addition of hemin yields a tetrameric DypB at $144 \mathrm{kDa}$. 